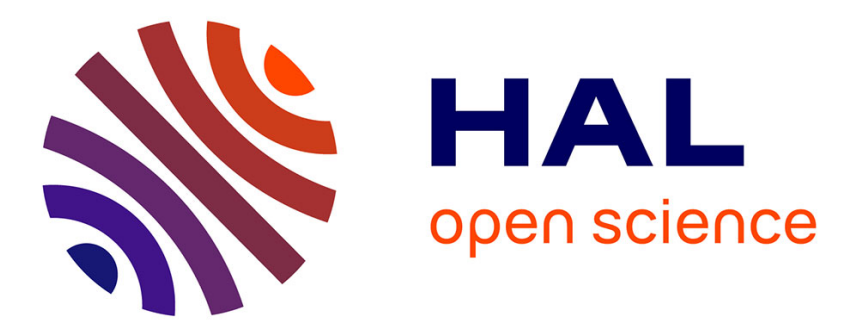

\title{
Growth of analytic functions in an ultrametric open disk and branched values
}

Kamal Boussaf, Alain Escassut

\section{To cite this version:}

Kamal Boussaf, Alain Escassut. Growth of analytic functions in an ultrametric open disk and branched values. 2019. hal-02358314v1

\section{HAL Id: hal-02358314 https://hal.uca.fr/hal-02358314v1}

Preprint submitted on 11 Nov 2019 (v1), last revised 8 Oct 2020 (v2)

HAL is a multi-disciplinary open access archive for the deposit and dissemination of scientific research documents, whether they are published or not. The documents may come from teaching and research institutions in France or abroad, or from public or private research centers.
L'archive ouverte pluridisciplinaire HAL, est destinée au dépôt et à la diffusion de documents scientifiques de niveau recherche, publiés ou non, émanant des établissements d'enseignement et de recherche français ou étrangers, des laboratoires publics ou privés. 


\title{
Growth of analytic functions in an ultrametric open disk and
}

\section{branched values}

\section{by Kamal Boussaf and Alain Escassut}

\begin{abstract}
Let $D$ be the open unit disk $|x|<R$ of a complete ultrametric algebraically closed field $\mathbb{K}$. We define the growth order $\rho(f)$, the growth type $\sigma(f)$ and the cotype $\psi(f)$ of an analytic function in $D$ and we show that, denoting by $q(f, r)$ the number of zeros of $f$ in the disk $|x| \leq r$ and putting $|f|(r)=\sup _{|x|<r}|f(x)|$, the infimum $\theta(f)$ of the $s$ such that $\lim _{r \rightarrow R^{-}} q(f, r)(R-r)^{s}=0$ satisfies $\theta(f)-1 \leq \rho(f) \leq \theta(f)$ and the infimum of the $s$ such that $\lim _{r \rightarrow R^{-}} \log (|f|(r))(R-r)^{s}=0$ is equal to $\rho(f)$. Moreover, if $\psi(f)<+\infty$, then $\theta(f)=\rho(f)$ and $\sigma(f)=0$. In zero residue characteristic, then $\rho\left(f^{\prime}\right)=\rho(f), \sigma\left(f^{\prime}\right)=\sigma(f)$, $\psi\left(f^{\prime}\right)=\psi(f)$. Suppose $\mathbb{K}$ has zero characteristic. Consider two unbounded analytic functions $f, g$ in $D$. If $\rho(f) \neq \rho(g)$, then $\frac{f}{g}$ has at most two perfectly branched values and if $\rho(f)=\rho(g)$ but $\sigma(f) \neq \sigma(g)$, then $\frac{f}{g}$ has at most three perfectly branched values; moreover, if $2 \sigma(g)<\sigma(f)$, then $\frac{f}{g}$ has at most two perfectly branched values.
\end{abstract}

Subject Classification: 12J25; 30D35; 30G06

Keywords: P-adic analytic functions in disks, order, type and cotype of growth, branched values.

\section{Introduction and main theorems}

Let $\mathbb{K}$ be an algebraically closed field complete with respect to an ultrametric absolute value. In [2] and [3] we defined the order of growth and the type of growth for entire functions in $\mathbb{K}$ in a similar way as it is known for complex entire functions [11] and we also defined a cotype of growth strongly linked to the order and the type: in most of the cases the cotype is the product of the order of growth by the type of growth.

Here we consider analytic functions in an "open" disk.

Notations and definitions: For every $r>0$, we denote by $d\left(0, r^{-}\right)$the disk $\{x \in$ $\mathbb{K}|| x \mid<r\}$ and by $d(0, r)$ the disk $\{x \in \mathbb{K}|| x \mid \leq r\}$. Throughout the paper we put $D=d\left(0, R^{-}\right)$. We denote by $\mathcal{A}(D)$ the $\mathbb{K}$-algebra of analytic functions in $D$ i.e. the power series $\sum_{n=0}^{\infty} a_{n} x^{n}$ converging in the disk $D$, which are the power series such that $\limsup _{n \rightarrow+\infty} \sqrt[n]{\left|a_{n}\right|} \leq \frac{1}{R}$, and we denote by $\mathcal{M}(R)$ the field of fractions of $\mathcal{A}(D)$ also called the meromorphic functions in $D$ [1], [7], [8], [10]. 
Let $f=\sum_{n=0}^{\infty} a_{n} x^{n} \in \mathcal{A}(D)$. Given $\left.r \in\right] 0, R\left[\right.$, we put $|f|(r)=\sup _{n \in \mathbb{N}}\left\{\left|a_{n}\right| r^{n}\right\}$. Then we know that $|f|(r)=\sup _{|x|=r}|f(x)|$, that $\mid$. | is an ultrametric absolute value on $\mathcal{A}(D)$ and that a function $f \in \mathcal{A}(D)$ is unbounded if and only if $\lim _{r \rightarrow R^{-}}|f|(r)=+\infty$ [7], [8].

We denote by log the Neperian Logarithm. In order to define a growth order similarly as it was done in the algebra of entire functions in $\mathbb{K}[2]$, [3], we can define in $\mathcal{A}(D)$ a growth order in the following way: given $r \in] 0, R[$, as it was done in complex analysis [5], [6], [9] we put $\rho(f, r)=\frac{\log (\log (|f|(r)))}{-\log (R-r)}$ and $\rho(f)=\limsup _{r \rightarrow R^{-}} \rho(f, r)$, hence $\rho(f)=$ $\limsup _{r \rightarrow R^{-}} \frac{\log (\log (|f|(r)))}{-\log (R-r)}$. Then $\rho(f)$ is called the order of growth of $f$.

On the other hand, for every $r \in] 0, R[$, we denote by $q(f, r)$ the number of zeros of $f$ in $d(0, r)$, taking multiplicity into account. If the set of the $s>0$ such that $\lim _{r \rightarrow R^{-}} q(f, r)(R-r)^{s}=0$ is empty, we put $\theta(f)=+\infty$. Else, we then denote by $\theta(f)$ the lowest bound of the $s>0$ such that $\lim _{r \rightarrow R^{-}} q(f, r)(R-r)^{s}=0$. Similarly, if the set of the $s>0$ such that $\lim _{r \rightarrow R^{-}} \log (|f|(r))(R-r)^{s}=0$ is empty, we put $\lambda(f)=+\infty$. Else, we denote by $\lambda(f)$ the lowest bound of the $s>0$ such that $\lim _{r \rightarrow R^{-}} \log (|f|(r))(R-r)^{s}=0$.

And if $0<\rho(f)<+\infty$, we put $\sigma(f, r)=\log (|f|(r))(R-r)^{\rho(f)}, \sigma(f)=\limsup _{r \rightarrow R^{-}} \sigma(f, r)$, $\psi(f, r)=q(f, r)(R-r)^{\rho(f)}$ and $\psi(f)=\limsup _{r \rightarrow R^{-}} \psi(f, r)$. We call $\sigma(f)$ the type of growth of $f$ and $\psi(f)$ the cotype of growth of $f$.

Let us recall that, as far as ultrametric entire functions are concerned, the order of growth is equal to the lowest bound of the $s>0$ such that $\lim _{r \rightarrow+\infty} \frac{\log (|f|(r))}{r^{s}}=0$ and to the lowest bound of the $s>0$ such that $\lim _{r \rightarrow+\infty} \frac{q(f, r)}{r^{s}}=0$. Here we will try to prove similar results. This paper is aimed at showing relations between these expressions $\rho(f), \sigma(f), \psi(f)$.

Notation: We will denote by $\mathcal{A}^{0}(D)$ the set of functions $f \in \mathcal{A}(D)$ such that $0<\rho(f)<$ $+\infty$.

Remark 1: Let $f \in \mathcal{A}(D)$ be bounded in $D$. Then $\rho(f)=0$.

Theorems 1 and 2 are easy and don't need any proof:

Theorem 1: Let $f, g \in \mathcal{A}(D)$. Then $\rho(f+g) \leq \max (\rho(f), \rho(g))$ and $\rho(f g)=\max (\rho(f), \rho(g))$.

Corollary 1.1: Let $f, g \in \mathcal{A}(D)$. Then $\rho\left(f^{n}\right)=\rho(f) \forall n \in \mathbb{N}^{*}$. If $\rho(f)>\rho(g)$, then $\rho(f+g)=\rho(f)$.

Theorem 2: Let $f \in \mathcal{A}(D)$ and let $P \in \mathbb{K}[x]$ be non-constant. Then $\rho(P \circ f)=\rho(f)$. 
Theorem 3: Let $f, g \in \mathcal{A}^{0}(D)$. Then $\psi(f g) \leq \psi(f)+\psi(g)$. Moreover, if $\rho(f)=\rho(g)$ then $\max (\psi(f), \psi(g)) \leq \psi(f g)$.

Remark 2: Let $f \in \mathcal{A}^{0}(D)$. If $s>\theta(f)$, then by definition, $\lim _{r \rightarrow R^{-}} q(f, r)(R-r)^{s}=0$. But if $s<\theta(f)$ then $\lim \sup q(f, r)(R-r)^{s}=+\infty$ because if lim $\sup q(f, r)(R-r)^{s}<+\infty$, $r \rightarrow R^{-}$

we can find $\left.s^{\prime} \in\right] s, \theta(f)\left[\right.$ and then we can check that $\lim _{r \rightarrow R^{-}} q(f, r)(R-r)^{s^{\prime}}=0$, a contradiction.

Thanks to the classical inequality $\left|f^{\prime}\right|(r) \leq \frac{|f|(r)}{r}[7]$, the following Theorem 4 is then immediate:

Theorem 4: Suppose $\mathbb{K}$ has characteristic 0. Let $f \in \mathcal{A}(D)$. Then $\rho\left(f^{\prime}\right) \leq \rho(f)$.

Remark 3: In a field of characteristic $p \neq 0$, certain analytic functions have a null derivative. This is why we must suppose that $\mathbb{K}$ has characteristic 0 in all statement involving derivatives.

In complex analysis, many estimates were given concerning the growth order of solutions of linear differential equations [5], [6], [9]. Here, by Corollary 1.1 and Theorem 4 we can immediately obtain Corollary 4.1 which is similar but more general than Theorem $\mathrm{C}$ in $[5]$ :

Corollary 4.1: $\quad$ Suppose $\mathbb{K}$ has characteristic 0. Consider the differential equation

$$
(\mathcal{E}) f^{(n)}+a_{n-1}(x) f^{(n-1)}(x)+\ldots+a_{0}(x) f(x)=0
$$

with $a_{j} \in \mathcal{A}^{0}(D), j=0, \ldots, n-1$ and $\rho\left(a_{j}\right)<\rho\left(a_{0}\right) \forall j=1, \ldots, n-1$. Then every non-trivial solution $f$ of $(\mathcal{E})$ satisfies $\rho(f) \geq \rho\left(a_{0}\right)$.

Theorem 5: Let $f \in \mathcal{A}(D)$. Then $\lambda(f)=\rho(f)$.

Remark 4: Similar results to Corollary 4.1 and Theorem 5 hold for entire functions thanks to results of [2] but were not stated in [2] and [3].

Theorem 6: Let $f, g \in \mathcal{A}^{0}(D)$. Then $\sigma(f g) \leq \sigma(f)+\sigma(g)$. If $\rho(f) \geq \rho(g)$, then $\sigma(f) \leq \sigma(f g)$. If $\rho(f)=\rho(g)$, then $\max (\sigma(f), \sigma(g)) \leq \sigma(f g)$.

If $\rho(f)=\rho(g)$ and $\sigma(f)>\sigma(g)$ then $\sigma(f+g) \geq \sigma(f)$. If $\rho(f+g)=\rho(f) \geq \rho(g)$ then $\sigma(f+g) \leq \max (\sigma(f), \sigma(g))$.

Corollary 6.1: Let $f, g \in \mathcal{A}^{0}(D)$ be such that $\rho(f) \neq \rho(g)$. Then

$$
\sigma(f+g) \leq \max (\sigma(f), \sigma(g))
$$


Theorem 7: Let $f \in \mathcal{A}(D)$. Then $\rho(f)=+\infty$ if and only if $\theta(f)=+\infty$. If $\rho(f)<+\infty$, then $\theta(f)-1 \leq \rho(f) \leq \theta(f)$. Moreover, if $\psi(f)<\infty$, then $\rho(f)=\theta(f)$.

Theorem 7 obviously suggests the following conjecture:

Conjecture: Let $f \in \mathcal{A}(D)$. Then $\rho(f)=\theta(f)$.

Theorem 8: $\quad$ Suppose $\mathbb{I K}$ has residue characteristic 0 . Then for every $f \in \mathcal{A}(D)$ we have $\rho\left(f^{\prime}\right)=\rho(f), \theta\left(f^{\prime}\right)=\theta(f)$. Moreover, if $\rho(f)<+\infty$, then $\sigma\left(f^{\prime}\right)=\sigma(f)$ and $\psi\left(f^{\prime}\right)=\psi(f)$.

Remark 5: Theorem 8 does not hold in residue characteristic $p>0$ because there exist functions $f \in \mathcal{A}(D)$ such that $\rho(f)>0$ and that $f^{\prime}$ is bounded, as shows the following example with $R=1: g(x)=\sum_{m=0}^{\infty} \frac{x^{p^{m}}}{p^{m}}$. We can see that $g^{\prime}(x)=\sum_{n=0}^{\infty} x^{p^{m}-1}$ hence $g^{\prime}$ is bounded and therefore $\rho\left(g^{\prime}\right)=0$. However, consider the sequence $\left(r_{m}\right)_{m \in \mathbb{N}}$ defined as $r_{m}=1-\frac{1}{p^{m}}$. We can check that $|g|\left(r_{m}\right) \geq p^{m}\left(r_{m}\right)^{p^{m}}$, hence

$$
\log \left(|g|\left(r_{m}\right)\right) \geq m+p^{m} \log \left(r_{m}\right)=m+p^{m} \log \left(1-\frac{1}{p^{m}}\right)
$$

When $m$ is big enough, we have $\log \left(1-\frac{1}{p^{m}}\right) \geq \frac{-2}{p^{m}}$, hence

$$
\log \left(|g|\left(r_{m}\right)\right) \geq m-p^{m}\left(\frac{2}{p^{m}}\right)=m-2 .
$$

Therefore, when $m$ is big enough, we have

$$
\frac{\log \left(\log \left(|g|\left(r_{m}\right)\right)\right)}{-\log \left(r_{m}\right)} \geq \frac{\log (m-2)}{-\log \left(1-\frac{1}{p^{m}}\right)}>\frac{\log (m-2)}{\frac{2}{p^{m}}}=\frac{p^{m}}{2} \log (m-2) .
$$

Thus, we have $\rho(g)=+\infty$.

Remark 6: Theorem 8 applies for instance to the complex Levi-Civita field whose residue characteristic is $0[12]$.

In [4], relations were examined between growth of entire functions and perfectly branched values. Now, we can look at possible similar relations.

Definition: Let $f \in \mathcal{M}(D)$. A value $b \in \mathbb{K}$ is called perfectly branched value for $f$ if all zeros of $f-b$ but finitely many are multiple.

Now, we must recall the notations of Nevanlinna's functions.

Notation: Let $f \in \mathcal{M}(D)$ be such that $f(0) \neq 0$ and $f(0) \neq \infty$. We denote by $Z(r, f)$ the counting function of zeros of $f$ in $D$ in the following way. Let $\left(a_{n}\right), 1 \leq n \leq u(r)$ be the finite sequence of zeros of $f$ such that $0<\left|a_{n}\right| \leq r$, of respective order $s_{n}$. 
We set $Z(r, f)=\sum_{n=1}^{u(r)} s_{n}\left(\log r-\log \left|a_{n}\right|\right)$ and so, $Z(r, f)$ is called the counting function of zeros of $f$ in $D$, counting multiplicity.

Next, we denote by $\bar{Z}(r, f)$ the counting function of zeros of $f$ without multiplicity: $\bar{Z}(r, f)=\sum_{n=1}^{u(r)}\left(\log r-\log \left|a_{n}\right|\right)$ and so, $\bar{Z}(r, f)$ is called the counting function of zeros of $f$ in D ignoring multiplicity.

In the same way, we put $N(r, f)=Z\left(r, \frac{1}{f}\right)$ and $\bar{N}(r, f)=\bar{Z}\left(r, \frac{1}{f}\right)$.

Now we can define the characteristic Nevanlinna function of $f$ in $] 0, R[$ as $T(r, f)=$ $\max (Z(r, f), N(r, f))$.

Remark 7: If we change the origin, the functions $Z, N, T$ are not changed, up to an additive constant.

Notation: We denote by $\mathcal{A}_{u}(D)$ the set of functions $f \in \mathcal{A}(D)$ that are unbounded in $D$.

Theorem 9 was already stated in Theorem 3 of [4]. Here we just complete the proof.

Theorem 9: $\quad$ Suppose $\mathbb{I K}$ has characteristic 0. Let $f, g \in \mathcal{A}(D)$ with $f \in \mathcal{A}_{u}(D)$ be such that $\limsup _{r \rightarrow R^{-}} \frac{T(r, f)}{T(r, g)}>2$. Then both $\frac{f}{g}$ and $\frac{g}{f}$ have at most two perfectly branched values.

Remark 8: If $f \in \mathcal{A}_{u}(D)$ and if $g \in \mathcal{A}(D)$ is bounded in $D$, then $\frac{f}{g}$ has at most two perfectly branched values.

We can easily obtain Theorem 10:

Theorem 10: Suppose $\mathbb{I K}$ has characteristic 0. Let $f, g \in \mathcal{A}_{u}(D)$ be such that $\rho(f)>$ $\rho(g)$. Then

$$
\liminf _{r \rightarrow R^{-}} \frac{T(r, g)}{T(r, f)}=0
$$

By Theorem 10 and Remark 8, we can now derive Corollary 10.1:

Corollary 10.1: Let $f, g \in \mathcal{A}(D)$ be such that $\rho(f) \neq \rho(g)$. Then both $\frac{f}{g}$ and $\frac{g}{f}$ have at most two perfectly branched values.

Now, when $\rho(f)=\rho(g)$, we can still give some precision. 
Theorem 11: Suppose $\mathbb{K}$ has characteristic 0. Let $f, g \in \mathcal{A}_{u}(D)$ and suppose that $\rho(f)=\rho(g) \in] 0,+\infty\left[\right.$ and $\sigma(f) \neq \sigma(g)$. Then both $\frac{f}{g}$ and $\frac{g}{f}$ have at most three perfectly branched values. Moreover, if $2 \sigma(g)<\sigma(f)$ or if $2 \sigma(f)<\sigma(g)$ then $\frac{f}{g}$ and $\frac{g}{f}$ have at most two perfectly branched values.

By Theorems 10 and 11, we can derive Corollary 11.1:

Corollary 11.1: Suppose $\mathbb{K}$ has characteristic 0. Let $f, g \in \mathcal{A}_{u}(D)$ be such that $\frac{f}{g}$ admits four distinct perfectly branched values. Then $\rho(f)=\rho(g)$. Moreover, if $\rho(f) \in$ ] $0,+\infty[$, then $\sigma(f)=\sigma(g)$.

\section{The proofs}

The proofs of Theorems 1 and 2 are immediate. The proof of Theorem 4 , is also immediate thanks to the property $\left|f^{\prime}\right|(r) \leq \frac{|f|(r)}{r}$ for functions having a zero at 0 , what we can suppose without loss of generality [7], [8].

In the proofs of Theorem 3,5, 6, 7, 8 we can suppose that $R$ belongs to $|\mathbb{K}|$ by sending our ground field into a field admitting a value group equal to $\mathbb{R}_{+}$. Therefore we can assume $R=1$ without loss of generality, through an obvious change of variable.

Proof of Theorem 3. Set $\rho(f)=s, \rho(g)=t$. Without loss of generality we can assume $s \geq t$. By Theorem 1, we have $\rho(f . g)=\rho(f)=s$. Now, for each $r>0$, we have $q(f . g, r)=q(f, r)+q(g, r)$ hence

$\psi(f g)=\limsup _{r \rightarrow R^{-}}(q(f, r)+q(g, r))(R-r)^{s} \leq \limsup _{r \rightarrow R^{-}} q(f, r)(R-r)^{s}+\limsup _{r \rightarrow R^{-}} q(g, r)(R-r)^{t}$

hence $\psi(f g) \leq \psi(f)+\psi(g)$.

Now, suppose $s=t$. Then

$$
\begin{gathered}
\psi(f g)=\limsup _{r \rightarrow R^{-}}(q(f, r)+q(g, r))(R-r)^{s} \geq \limsup _{r \rightarrow R^{-}} \max (q(f, r), q(g, r))(R-r)^{s} \\
=\max (\psi(f), \psi(g)),
\end{gathered}
$$

which ends the proof.

Proof of Theorem 5: First we will prove that $\rho(f) \leq \lambda(f)$. Obviously, we can assume that $\lambda(f)<+\infty$. Let $s$ be such that $\lim _{r \rightarrow R^{-}} \log (|f|(r))(R-r)^{s}=0$. Let us fix $\epsilon>0$. For $r$ close enough to $R$, we have $\log (|f|(r))(R-r)^{s} \leq \epsilon$, hence $\log (|f|(r)) \leq \frac{\epsilon}{(R-r)^{s}}$, therefore 
$\log (\log (|f|(r))) \leq \log \epsilon-s \log (R-r)$ hence

$$
\frac{\log (\log (|f|(r)))}{(-\log (R-r))} \leq \frac{\log (\epsilon)}{(-\log (R-r))}+s
$$

and hence

$$
\limsup _{r \rightarrow R^{-}} \frac{\log (\log (|f|(r)))}{(-\log (R-r))} \leq s
$$

i.e. $\rho(f) \leq s$. This is true for every $s$ such that $\lim _{r \rightarrow R^{-}} \log (|f|(r))(R-r)^{s}=0$ and hence $\rho(f) \leq \lambda(f)$.

On the other hand, we notice that, by definition of $\lambda(f)$, either $\lambda(f)=0$ and then $\lambda(f) \leq \rho(f)$, or

$$
\lambda(f)=\sup \{s \in] 0,+\infty\left[\mid \limsup _{r \rightarrow R^{-}} \log (|f|(r))(R-r)^{s}>0\right\} .
$$

Thus, suppose that $\lambda(f)>0$. Let us take $s \in] 0, \lambda(f)[$. We have a number $b>0$ such that

$$
\limsup _{r \rightarrow R^{-}}\left(\log \left(|f|(r)(R-r)^{s}\right) \geq b>0\right.
$$

Let us fix $\epsilon \in] 0, b\left[\right.$. There exists a sequence $\left(r_{n}\right)_{n \in \mathbb{N}}$ in $] 0, R\left[\right.$ such that $\lim _{n \rightarrow+\infty} r_{n}=R$ and such that, when $n$ is big enough, we have $b-\epsilon \leq \log \left(|f|\left(r_{n}\right)\right)\left(R-r_{n}\right)^{s}$, hence $-s \log \left(R-r_{n}\right)+\log (b-\epsilon)<\log \left(\log \left(|f|\left(r_{n}\right)\right)\right)$ therefore

$$
s+\frac{\log (b-\epsilon)}{\left(-\log \left(R-r_{n}\right)\right)} \leq \frac{\log \left(\operatorname { l o g } \left(|f|\left(r_{n}\right)\right.\right.}{\left(-\log \left(R-r_{n}\right)\right)} .
$$

Consequently, $\limsup _{n \rightarrow+\infty} \frac{\log \left(\log \left(|f|\left(r_{n}\right)\right)\right.}{\left(-\log \left(R-r_{n}\right)\right)} \geq s$, therefore $\rho(f) \geq s$. But this holds for every $s<\lambda(f)$. Thus, $\rho(f) \geq \lambda(f)$ and finally, $\rho(f)=\lambda(f)$.

Proof of Theorem 6: Let $s=\rho(f), t=\rho(g)$ and suppose $s \geq t$. When $r$ is close enough to $R$, we have $\max (\log (|f|(r)), \log (|g|(r)) \leq \log (|f \cdot g|(r))=\log (|f|(r))+\log (|g|(r))$ and by Theorem 1, we have $\rho(f g)=s$. Therefore

$$
\begin{gathered}
\sigma(f g)=\limsup _{r \rightarrow R^{-}}\left(\log (|f \cdot g|(r))(R-r)^{s}\right) \\
\leq \limsup _{r \rightarrow R^{-}}\left(\log (|f|(r))(R-r)^{s}\right)+\limsup _{r \rightarrow R^{-}}\left(\log (|g|(r))(R-r)^{t}\right) \\
\leq \limsup _{r \rightarrow R^{-}}\left(\log (|f|(r))(R-r)^{s}\right)+\limsup _{r \rightarrow R^{-}}\left(\log (|g|(r))(R-r)^{t}=\sigma(f)+\sigma(g) .\right.
\end{gathered}
$$

On the other hand,

$$
\sigma(f)=\limsup _{r \rightarrow R^{-}} \log (|f|(r))(R-r)^{s} \leq \limsup _{r \rightarrow+R^{-}}\left(\log (|f g|(r))(R-r)^{s} .\right.
$$


But $\rho(f g)=s$, hence $\sigma(f) \leq \sigma(f g)$. Particularly, if $\rho(f)=\rho(g)$, then $\max (\sigma(f), \sigma(g)) \leq$ $\sigma(f g)$.

Now, suppose again that $\rho(f)=\rho(g)=s$ and suppose $\sigma(f)>\sigma(g)$. Let $s=\rho(f), b=$ $\sigma(f)$. Then $b>0$. Let $\left(r_{n}\right)_{n \in \mathbb{N}}$ be a sequence such that $\lim _{n \rightarrow+\infty} r_{n}=R$ and

$\lim _{n \rightarrow+\infty}\left(\log \left(|f|\left(r_{n}\right)\right)\left(R-r_{n}\right)^{s}\right)=b$. Since $\sigma(g)<\sigma(f)$, we notice that when $n$ is big enough we have $|g|\left(r_{n}\right)<|f|\left(r_{n}\right)$. Consequently, when $n$ is big enough, we have $|f+g|\left(r_{n}\right)=|f|\left(r_{n}\right)$ and hence

$$
\left.\lim _{n \rightarrow+\infty}\left(\log \left(|f+g|\left(r_{n}\right)\right)\right)\left(R-r_{n}\right)^{s}\right)=b
$$

By definition of $\sigma$ we have $\sigma(f+g) \geq \lim _{n \rightarrow+\infty}\left(\log \left(|f+g|\left(r_{n}\right)\right)\right)\left(R-r_{n}\right)^{\rho(f+g)}$. By Theorem 1 , we have $\rho(f+g) \leq s$, hence

$$
\begin{gathered}
\sigma(f+g) \geq \lim _{n \rightarrow+\infty}\left(\log \left(|f+g|\left(r_{n}\right)\right)\right)\left(R-r_{n}\right)^{\rho(f+g)} \geq \lim _{n \rightarrow+\infty}\left(\log \left(|f+g|\left(r_{n}\right)\right)\right)\left(R-r_{n}\right)^{s} \\
=\lim _{n \rightarrow+\infty} \log \left(|f|\left(r_{n}\right)\right)\left(R-r_{n}\right)^{s}=\sigma(f)
\end{gathered}
$$

therefore by $(1), \sigma(f+g) \geq \sigma(f)$.

Finally, suppose now that $\rho(f+g)=\rho(f) \geq \rho(g)$. Let $s=\rho(f)$ and $t=\rho(g)$. Then,

$$
\begin{gathered}
\sigma(f+g)=\limsup _{r \rightarrow R^{-}}(\log (|f+g|(r)))(R-r)^{s} \\
\leq \max \left(\limsup _{r \rightarrow R^{-}}(\log (|f|(r)))(R-r)^{s}, \limsup _{r \rightarrow R^{-}}(\log (|g|(r)))(R-r)^{s}\right) \\
\max \left(\limsup _{r \rightarrow R^{-}}(\log (|f|(r)))(R-r)^{s}, \limsup _{r \rightarrow R^{-}}(\log (|g|(r)))(R-r)^{t}\right)=\max (\sigma(f), \sigma(g))
\end{gathered}
$$

which ends the proof.

In the proof of Theorem 10, we will use the following Lemma $G$ and in the proof of of Theorem 9 we will use the following Lemmas J:

Lemma G: Let $f \in \mathcal{A}(D)$. The following three statements are equivalent:

1) $f \in \mathcal{A}_{u}(D)$,

2) $\lim _{r \rightarrow R^{-}}|f|(r)=+\infty$

3) $\lim _{r \rightarrow R^{-}} T(r, f)=+\infty$.

Lemma J: Let $\phi=\frac{f}{g} \in \mathcal{M}(D)$ with $f, g \in \mathcal{A}(D)$ having no common zero. Then, given $b \in \mathbb{K}$, we have $Z(r, \phi-b)=Z(r, f-b g) \leq \max (T(r, f), T(r, g))+O(1)=T(r, \phi)+O(1)$.

In the proof of Theorem 7 we will need the basic Lemma L due to the concavity of Logarithm and the classical Theorem T ( Corollary 22.27 in [8]): 
Lemma L: Let $a \in[1,+\infty[$ and $b \in[0,+\infty[$. Then $\log (a+b) \leq \log (a)+\log (b+1)$.

Proof: Indeed, since $a \geq 1$, we have $\log (a+b) \leq \log (a(b+1))=\log (a)+\log (b+1)$.

Theorem T: Let $f \in \mathcal{A}(D)$ be such that $f(0) \neq 0$ and let $\left(a_{n}\right)_{n \in \mathbb{N}}$ be the sequence of zeros of $f$, of respective multiplicity order $w_{n}$, with $\left|a_{n}\right| \leq\left|a_{n+1}\right|$. Let $c_{n}=\left|a_{n}\right|, n \in \mathbb{N}$. Suppose $c_{n} \leq r<c_{n+1}$. Then

$$
\log \left(|f|(r)=\log |f(0)|+\sum_{n=0}^{k} w_{n}\left(\log (r)-\log \left(c_{n}\right)\right)\right.
$$

Corollary T.1: Let $f \in \mathcal{A}(D)$ and let $\left.r_{1}, r_{2} \in\right] 0, R\left[\right.$ with $r_{1}<r_{2}$. Then

$$
\left(\frac{r_{2}}{r_{1}}\right)^{q\left(f, r_{1}\right)} \leq \frac{|f|\left(r_{2}\right)}{|f|\left(r_{1}\right)} \leq\left(\frac{r_{2}}{r_{1}}\right)^{q\left(f, r_{2}\right)} .
$$

Proof of Theorem 7: We will denote by $|\cdot|_{\infty}$ the Archimedean absolute value of $\mathbb{R}$. Let us first choose $s>\theta(f)$. Then $\lim _{r \rightarrow R^{-}} q(f, r)(R-r)^{s}=0$. Now, since $\lim _{r \rightarrow R^{-}}|f|(r)=+\infty$, we can take $\ell \in] 0, R[$ such that $|f|(\ell)>1$. Then we can take $b>0$ such that

$$
q(f, r) \leq b(R-r)^{-s} \forall r \in[\ell, R[.
$$

Now, taking $r \in\left[\ell, R[\right.$, by Theorem $\mathrm{T}$, we have $\log (|f|(r)) \leq \log (|f|(\ell)))+q(f, r)\left(\log \left(\frac{r}{\ell}\right)\right)$ which leads to

$$
\log (|f|(r)) \leq \log (|f|(\ell)))+b(R-r)^{-s}\left(\log \left(\frac{r}{\ell}\right)\right)
$$

hence

$$
\left.\log (\log (|f|(r))) \leq \log (\log (|f|(\ell)))+b(R-r)^{-s}\left(\log \left(\frac{r}{\ell}\right)\right)\right)
$$

therefore, by Lemma L, we can derive

$$
\log (\log (|f|(r))) \leq \log (\log (|f|(\ell)))+\log \left(b(R-r)^{-s}\left(\log \left(\frac{r}{\ell}\right)\right)+1\right) .
$$

Now, since $s>0$, there obviously exists $h \in\left[\ell, R\left[\right.\right.$ such that $b(R-r)^{-s} \geq 1 \forall r \in\left[h, R^{-}[\right.$, therefore by Lemma L again,

$$
\log (\log (|f|(r))) \leq \log (\log (|f|(\ell)))+\log \left(b(R-r)^{-s}\left(\log \left(\frac{r}{\ell}\right)\right)+\log (1+1)\right.
$$

i.e.

(2) $\quad \log (\log (|f|(r))) \leq \log (\log (|f|(\ell)))+\log (b)-s \log (R-r)+\log \left(\left(\log \left(\frac{r}{\ell}\right)\right)+\log (2)\right.$ 
Consequently, by (2), we obtain

$$
\frac{\log (\log (|f|(r)))}{-\log (R-r)} \leq \frac{\log (\log (|f|(\ell)))}{-\log (R-r)}+\frac{\log (b)}{-\log (R-r)}+s+\frac{\log \left(\log \left(\frac{r}{\ell}\right)\right)+\log (2)}{-\log (R-r)} .
$$

We can check that

$$
\lim _{r \rightarrow R^{-}} \frac{\log (\log (|f|(\ell)))+\log (b)}{-\log (R-r)}=\lim _{r \rightarrow R^{-}} \frac{\log \left(\log \left(\frac{r}{\ell}\right)+\log (2)\right.}{-\log (R-r)}=0
$$

and hence $\limsup _{r \rightarrow R^{-}} \frac{\log (\log (|f|(r)))}{-\log (R-r)} \leq s$. Consequently, choosing $\epsilon>0$, there exists $u \in[\ell, 1[$ such that $\frac{\log (\log (|f|(r)))}{-\log (R-r)} \leq s+\epsilon \forall r \in[u, R[$ and hence $\rho(f) \leq s+\epsilon$. But since that holds for every $s>\theta(f)$ and for every $\epsilon>0$, we have $\rho(f) \leq s$ and hence $\rho(f) \leq \theta(f)$.

Let us now show that $\rho(f) \geq \theta(f)-1$. By Corollary T.1, we have

(3) $\log (|f|(r))-\log \left(|f|\left(\frac{r^{2}}{R}\right)\right) \geq q\left(f, \frac{r^{2}}{R}\right)\left(\log (r)-\log \left(\frac{r^{2}}{R}\right)\right)=q\left(f, \frac{r^{2}}{R}\right)(\log (R)-\log (r))$.

Consider now a number $s<\theta(f)$ and a sequence $\left(r_{n}\right)_{n \in \mathbb{N}}$ of $] 0, R\left[\right.$ such that $\lim _{n \rightarrow+\infty} r_{n}=R$ and such that $\limsup _{n \rightarrow+\infty} q\left(f, r_{n}\right)\left(R-r_{n}\right)^{s} \geq b>0$. Then by (3) we have

$$
\log \left(|f|\left(r_{n}\right)\right) \geq \frac{b\left(\log (R)-\log \left(r_{n}\right)\right)}{\left(R-\frac{r_{n}^{2}}{R}\right)^{s}}
$$

Consequently,

$\left.\log \left(\log \left(|f|\left(r_{n}\right)\right)\right) \geq \log (b)+\log \left(\log (R)-\log \left(r_{n}\right)\right)\right)-s\left(\log \left(R-r_{n}\right)+\log \left(R+r_{n}\right)\right)+2 s \log (R)$ and therefore

$\frac{\log \left(\log \left(|f|\left(r_{n}\right)\right)\right)}{-\log \left(R-r_{n}\right)} \geq \frac{\log (b)}{-\log \left(R-r_{n}\right)}+\frac{\log \left(\log (R)-\log \left(r_{n}\right)\right)}{-\log \left(R-r_{n}\right)}+s\left(1+\frac{\log \left(R+r_{n}\right)+2 \log (R)}{-\log \left(R-r_{n}\right)}\right)$.

Clearly,

$$
\lim _{n \rightarrow+\infty}\left(\frac{\log (b)}{\log \left(R-r_{n}\right)}\right)=\lim _{n \rightarrow+\infty} \frac{\log \left(R+r_{n}\right)+2 \log (R)}{\log \left(R-r_{n}\right)}=0
$$

and by elementary reasonings, we can check that

$$
\lim _{t \rightarrow R^{-}} \frac{\log (\log (R)-\log (t))}{\log (R-t)}=1,
$$

therefore 


$$
\lim _{n \rightarrow+\infty} \frac{\log \left(\log (R)-\log \left(r_{n}\right)\right)}{\log \left(R-r_{n}\right)}=1
$$

Consequently,

$$
\limsup _{n \rightarrow+\infty} \frac{\log \left(\log \left(|f|\left(r_{n}\right)\right)\right)}{-\log \left(R-r_{n}\right)} \geq s-1
$$

and therefore

$$
\limsup _{r \rightarrow R^{-}} \frac{\log (\log (|f|(r)))}{-\log (R-r)} \geq s-1 .
$$

That holds for every $s<\theta(f)$ and shows that if $\theta(f)<+\infty$, then $\rho(f) \geq \theta(f)-1$ and if $\theta(f)=+\infty$, then $\rho(f)=\theta(f)=+\infty$. Therefore, either $\rho(f)<+\infty$ and then $\rho(f) \geq \theta(f)-1$, or $\rho(f)=\theta(f)=+\infty$.

Let us now show that $\rho(f) \geq \theta(f)$ when $\psi(f)<+\infty$. Suppose $\theta(f)>\rho(f)$ and let $s \in] \rho(f), \theta(f)\left[\right.$. Then by Remark 2 we have $\limsup _{r \rightarrow R^{-}} q(f, r)(R-r)^{s}=+\infty$, but then $\limsup q(f, r)(R-r)^{\rho(f)}=+\infty$, i.e. $\psi(f)=+\infty$, a contradiction. Therefore $\theta(f) \leq \rho(f)$

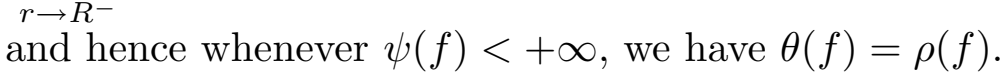

Proof of Theorem 8: Without loss of generality, we can assume that $f(0)=0$. Then, since $\mathbb{K}$ has residue characteristic 0 , we have $\left.q\left(f^{\prime}, r\right)=q(f, r)-1 \forall r \in\right] 0, R\left[\right.$ and $\left|f^{\prime}\right|(r)=$ $\left.\frac{|f|(r)}{r} \forall r \in\right] 0, R[[7],[8]$, and therefore we can easily check that

$$
\frac{\log (\log (|f|(r))-\log (r))}{-\log (R-r)}=\frac{\log \left(\log \left(\left|f^{\prime}\right|(r)\right)\right)}{-\log (R-r)}
$$

Now take $\epsilon>0$. There obviously exists $\left.r_{0} \in\right] 0, R[$ such that

$$
\log \left(|f|(r)-\epsilon \leq \log \left(|f|(r)-\log (r) \forall r \in\left[r_{0}, R[,\right.\right.\right.
$$

hence $\log \left(|f|(r)-\epsilon \leq \log \left(\left|f^{\prime}\right|(r) \leq \log \left(|f|(r) \forall r \in\left[r_{0}, R[\right.\right.\right.\right.$, therefore

$$
\frac{\log (\log (|f|(r))-\epsilon)}{-\log (R-r)}<\frac{\log \left(\log \left(\left|f^{\prime}\right|(r)\right)\right)}{-\log (R-r)}<\frac{\log (\log (|f|(r)))}{-\log (R-r)} .
$$

Consequently, since $\lim _{r \rightarrow R^{-}}|f|(r)=+\infty$, we have $\limsup _{r \rightarrow R^{-}} \rho\left(f^{\prime}, r\right)=\limsup _{r \rightarrow R^{-}} \rho(f, r)$, which shows that $\rho(f)=\rho\left(f^{\prime}\right)$.

Suppose now that $\rho(f)<+\infty$. We have

$$
\sigma\left(f^{\prime}, r\right)=(\log (|f|(r))-\log (r))(R-r)^{\rho(f)}=\sigma(f, r)-(\log (r))(R-r)^{\rho(f)}
$$

and then

$$
\limsup _{r \rightarrow R^{-}}(\log (|f|(r))-\log (r))(R-r)^{\rho(f)}=\limsup _{r \rightarrow R^{-}}(\log (|f|(r)))(R-r)^{\rho(f)}
$$


i.e. $\sigma\left(f^{\prime}\right)=\sigma(f)$. In the same way, then $\psi\left(f^{\prime}\right)=\psi(f)$.

From Theorem N2 [3] or Theorem 40.24 in [8], we can extract the following statement:

Theorem N [3], [8] Suppose $\mathbb{I}$ has characteristic 0 . Let $f \in \mathcal{M}(D)$ and let $b_{1}, \ldots, b_{q} \in$ IK. Then

$$
(q-1) T(r, f) \leq \sum_{j=1}^{q} \bar{Z}\left(r, f-b_{j}\right)+\bar{N}(r, f)+O(1)
$$

Proof of Theorem 9: Without loss of generality, we can place ourselves in an algebraically closed spherically complete extension to obtain the same conclusion because the Nevanlinna functions are the same in such an extension. Then, by Lazard's Theorem (Theorem 29.6 in [8]) we can suppose that $f$ and $g$ have no common zeros. Next, we can suppose that $f(0)=g(0) \neq 0$ and set $\phi=\frac{f}{g}$. We have $T(r, \phi)=\max (T(r, f), T(r, g))$. Now, by hypothesis, there exists $\gamma<\frac{1}{2}$ and a sequence $\left(r_{n}\right)_{n \in \mathbb{N}}$ in $] 0, R\left[\right.$ such that $\lim _{n \rightarrow+\infty} r_{n}=R$ and such that

$$
T\left(r_{n}, g\right) \leq \gamma T\left(r_{n}, f\right) \forall n \in \mathbb{N} \text {. }
$$

Suppose that $\phi$ has 3 perfectly branched values $b_{j}, j=1,2,3$. Applying Theorem $\mathrm{N}$ we have

$$
2 T(r, \phi) \leq \sum_{j=1}^{3} \bar{Z}\left(r, \phi-b_{j}\right)+\bar{N}(r, \phi)+O(1) .
$$

But here, for each $j=1,2,3$, we notice that $\bar{Z}\left(r, \phi-b_{j}\right) \leq \frac{Z\left(r, \phi-b_{j}\right)}{2}+q_{j} \log (r)$ with $q_{j} \in \mathbb{N}$ and by Lemma $\mathrm{J}, Z\left(r, \phi-b_{j}\right)=Z\left(r, f-b_{j} g\right) \leq \max (T(r, f), T(r, g))+O(1)$. But since $T\left(r_{n}, f\right)>T\left(r_{n}, g\right)$, we have $T\left(r_{n}, \phi-b_{j}\right) \leq T\left(r_{n}, f\right)+O(1)$, hence $\bar{Z}\left(r, \phi-b_{j}\right) \leq$ $\frac{T\left(r_{n}, f\right)}{2}+q_{j} \log \left(r_{n}\right)+O(1)$. Now, putting $q=q_{1}+q_{2}+q_{3}$, by (2) we obtain

$$
2 T\left(r_{n}, f\right) \leq \frac{3 T\left(r_{n}, f\right)}{2}+T\left(r_{n}, g\right)+q \log \left(r_{n}\right)+O(1)
$$

hence by (1),

$$
T\left(r_{n}, f\right) \leq\left(\frac{1}{2}+\gamma\right) T\left(r_{n}, f\right)+O(1)
$$

with $\gamma<\frac{1}{2}$ a contradiction since, by Lemma G, we have $\lim _{r \rightarrow R^{-}} T(r, f)=+\infty$. Similarly, considering $\frac{1}{\phi}$, we can see that $\frac{g}{f}$ has at most two branched values. 
Proof of Theorem 10: Let $\gamma=\frac{\rho(g)}{\rho(f)}$ and let $\left(r_{n}\right)_{n \in \mathbb{N}}$ be a sequence in $] 0, R[$ such that $\lim _{n \rightarrow+\infty} r_{n}=R$ and $\lim _{n \rightarrow+\infty} \frac{\log \left(\log \left(|f|\left(r_{n}\right)\right)\right)}{-\log \left(R-r_{n}\right)}=\rho(f)$. By hypothesis, we have

$$
\lim _{n \rightarrow+\infty} \frac{\log \left(\log \left(|g|\left(r_{n}\right)\right)\right)}{\log \left(\log \left(|f|\left(r_{n}\right)\right)\right)} \leq \gamma
$$

hence

$$
\lim _{n \rightarrow+\infty} \frac{T\left(r_{n}, g\right)}{T\left(r_{n}, f\right)} \leq \gamma
$$

Take $\beta \in] \gamma, 1[$. Then when $n$ is big enough, we can get

$$
\frac{T\left(r_{n}, g\right)}{T\left(r_{n}, f\right)} \leq\left(T\left(r_{n}, f\right)\right)^{\beta-1}
$$

But since $\beta<1$ and since, by Lemma $\mathrm{G}, \lim _{n \rightarrow \infty} T\left(r_{n}, f\right)=+\infty$, one sees that $\lim _{n \rightarrow \infty}\left(T\left(r_{n}, f\right)\right)^{\beta-1}=0$, which ends the proof.

Proof of Theorem 11: Without loss of generality, we can suppose that $\sigma(f)>\sigma(g)$ and, as explained in the proof of Theorem 9, we can suppose that $f$ and $g$ have no common zero and satisfy $f(0)=g(0) \neq 0$. Put $\phi=\frac{f}{g}$. Then we have $T(r, \phi)=$ $\max (T(r, f), T(r, g)), r>0$. Put $\rho(f)=t$. There exist $\gamma>0$ and a sequence $\left(r_{n}\right)_{n \in \mathbb{N}}$ in ] $0, R\left[\right.$ such that $\lim _{n \rightarrow+\infty} r_{n}=R$ and

$$
\log \left(|f|\left(r_{n}\right)\right)\left(R-r_{n}\right)^{t} \geq \log \left(|g|\left(r_{n}\right)\right)\left(R-r_{n}\right)^{t}+\gamma, \forall n \in \mathbb{N}
$$

hence

$$
\log \left(|f|\left(r_{n}\right)\right) \geq \gamma\left(R-r_{n}\right)^{-t}+\log \left(|g|\left(r_{n}\right)\right), \forall n \in \mathbb{N}
$$

consequently,

$$
T\left(r_{n}, f\right) \geq \gamma\left(R-r_{n}\right)^{-t}+T\left(r_{n}, g\right), \forall n \in \mathbb{N}
$$

Consequently,

$$
T\left(r_{n}, \phi\right)=T\left(r_{n}, f\right)
$$

when $n$ is big enough. Suppose now that $\phi$ admits 4 perfectly branched values $b_{j}, j=$ $1,2,3,4$ and let $q$ be the total number of zeros of order 1 of the $\phi-b_{j}, j=1,2,3,4$. Applying Theorem $\mathrm{N}$ to $\phi$, we have

$$
\left.3 T\left(r_{n}, \phi\right) \leq \sum_{j=1}^{4} \bar{Z}\left(r_{n}, \phi-b_{j}\right)+\bar{N}\left(r_{n}, \phi\right)\right)+O(1)
$$




$$
\leq \frac{4 T\left(r_{n}, f\right)}{2}+q \log \left(r_{n}\right)+T\left(r_{n}, g\right)+O(1)
$$

hence by (1),

$$
3 T\left(r_{n}, f\right) \leq 2 T\left(r_{n}, f\right)+T\left(r_{n}, f\right)+q \log \left(r_{n}\right)-\gamma\left(R-r_{n}\right)^{-t}+O(1)
$$

therefore

$$
3 T\left(r_{n}, f\right) \leq 2 T\left(r_{n}, f\right)+T\left(r_{n}, f\right)-\gamma\left(R-r_{n}\right)^{-t}+O(1)
$$

Clearly $\lim _{n \rightarrow+\infty}+q \log \left(r_{n}\right)-\gamma\left(R-r_{n}\right)^{-t}=-\infty$ and hence that inequality is absurd when $n$ is big enough, which ends the proof of the first claim.

Suppose now that $2 \sigma(g)<\sigma(f)$ and set $\beta=\frac{\sigma(f)}{2}-\sigma(g)$. So, there exists a sequence $\left(r_{n}\right)_{n \in \mathbb{N}}$ in $] 0, R\left[\right.$ such that $\lim _{n \rightarrow+\infty} r_{n}=R$ and

$$
2 T\left(r_{n}, g\right)\left(R-r_{n}\right)^{t}+2 \beta \leq T\left(r_{n}, f\right)\left(R-r_{n}\right)^{t}
$$

hence

$$
T\left(r_{n}, g\right) \leq \frac{T\left(r_{n}, f\right)}{2}-\beta\left(R-r_{n}\right)^{-t} \forall n \in \mathbb{N}
$$

Suppose now that $\phi$ has three perfectly branched values $b_{j}, j=1,2,3$. Similarly to (3), thanks to (4) now we can get

$$
\begin{gathered}
2 T\left(r_{n}, \phi\right)=2 T\left(r_{n}, f\right) \leq \sum_{j=1}^{3} \bar{Z}\left(r_{n}, \phi-b_{j}\right)+\bar{Z}\left(r_{n}, g\right)+O(1) \\
\left.\leq \frac{3 T\left(r_{n}, f\right)}{2}+\frac{T\left(r_{n}, f\right)}{2}-\beta\left(R-r_{n}\right)^{-t}+O(1)\right) .
\end{gathered}
$$

Since $\lim _{n \rightarrow+\infty}-\beta\left(R-r_{n}\right)^{-t}=-\infty$, we see a contradiction which finishes the proof.

\section{References:}

[1] Y. Amice, Les nombres p-adiques. P.U.F. (1975).

[2] K. Boussaf, A. Boutabaa, A. Escassut, Growth of p-adic entire functions and applications. Houston Journal of Mathematics, vol 40 (3), p.715-736 (2014).

[3] K. Boussaf, A. Boutabaa, A. Escassut, Order, type and cotype of growth for p-adic entire functions, a survey with additional properties. p-adic Numbers, Ultrametric Analysis and Applications to Number Theory, vol. 8, No. 4, pp. 280-297 (2016). 
[4] K. Boussaf, A. Escassut and J. Ojeda, Complex and p-adic branched functions and growth of entire functions, Bulletin of the Belgian Mathematical Society Simon Stevin 22, p.781-796, (2015).

[5] T.B. Cao, H.-X. Yi, The growth of solutions of linear differential equations with coefficients of iterated order in the unit disc. J. Math. Anal. Appl. 319 pp. 278-294 (2006).

[6] I. Chyzhykov, G. Gundersen, J. Heittokangas, Linear differential equations and logarithmic derivative estimates. Proc. London Math. Soc. 86 (3) p. 735-754 (2003).

[7] A. Escassut, Analytic Elements in p-adic Analysis. World Scientific Publishing Co. Pte. Ltd. Singapore, (1995).

[8] A. Escassut, Value Distribution in p-adic Analysis. World Scientific Publishing Co. Pte. Ltd. Singapore, (2015).

[9] J. Heittokangas, On complex differential equations in the unit disc. Ann. Acad. Sci. Fenn. Math. Diss. 122 p. 1-54 (2000).

[10] M. Krasner, Prolongement analytique uniforme et multiforme dans les corps valués complets. Les tendances géométriques en algèbre et théorie des nombres, ClermontFerrand, p.94-141 (1964). Centre National de la Recherche Scientifique (1966), (Colloques internationaux de C.N.R.S. Paris, 143).

[11] Lee A. Rubel, Entire and meromorphic functions. Springer-Verlag, New York, (1996).

[12] K. Shamseddine, A brief survey of the study of power series and analytic functions on the Levi-Civita fields, Contemporary Mathematics Volume 596, p. 269-279, (2013).

Laboratoire de Mathematiques Blaise Pascal,

UCA/CNRS-UMR6620

Campus des Czeaux

3 Place Vasarely

TSA60026 - CS60026

63178 AUBIERE CEDEX

FRANCE 